\title{
The Impact of Spousal Chronic Health Shock on Subjective Well-Being Among Elderly in China: An Urban-Rural Dimension
}

\section{Zhongliang Zhou}

Xi'an Jiaotong University

Dan Cao ( $\sim$ cdan1996@163.com)

Xi'an Jiaotong University https://orcid.org/0000-0002-8143-0998

\section{Yaxin Zhao}

Xi'an Jiaotong University

Dantong Zhao

Xi'an Jiaotong University

\section{Yangling Ren}

Xi'an Jiaotong University

\section{Rashed Nawaz}

Xi'an Jiaotong University

Jian Zhang

Xi'an Jiaotong University

\section{Chi Shen}

Xi'an Jiaotong University

Guanping Liu

Xi'an Jiaotong University

Xiaohui Zhai

Xi'an Jiaotong University

\section{Xiaojing Fan}

Xi'an Jiaotong University

Jianmin Gao

Xi'an Jiaotong University

\section{Research}

Keywords: spousal chronic health shock, elderly, subjective well-being, urban China, rural China

Posted Date: September 30th, 2020

DOl: https://doi.org/10.21203/rs.3.rs-81474/v1 
License: (c) (i) This work is licensed under a Creative Commons Attribution 4.0 International License. Read Full License 
1 The impact of spousal chronic health shock on subjective well-being among elderly in China: An

2 urban-rural dimension

3 Zhongliang Zhou ${ }^{1}$, Dan $\mathrm{Cao}^{1}$, Yaxin Zhao ${ }^{1}$, Dantong Zhao ${ }^{1}$, Yangling Ren ${ }^{1}$, Rashed Nawaz ${ }^{1}$, Jian Zhang ${ }^{1}$,

4 Chi Shen ${ }^{1}$, Guanping Liu ${ }^{1}$, Xiaohui Zhai ${ }^{1}$, Xiaojing Fan ${ }^{1}$, Jianmin Gao ${ }^{1}$

$5 \quad \quad{ }^{1}$ School of Public Policy and Administration, Xi'an Jiaotong University, PR China.

6 Corresponding author: Dan Cao, School of Public Policy and Administration, Xi'an Jiaotong

7 University, PR China.

$8 \quad$ Tel.: +86-13992028934, E-mail: cdan1996@163.com

9 


\section{Abstract}

\section{Backgrounds}

3 Chronic conditions could bring not only heavy economic burden on families, but also had negative

4 emotional and mental impacts to patients and their family members. The aim of this study is to explore 5 the effect of chronic health shock of elderly people on spousal subjective well-being in China from 6 urban-rural dimension.

\section{$7 \quad$ Methods}

8 We used two most recent databases — 2011 and 2015 — of China Health and Nutrition Survey, and the 9 total sample were categorized into urban sample and rural sample. Participants were defined as treatment group if his/her spouse was diagnosed with chronic disease in 2015 and not diagnosed in 2011; others were defined as control group. Propensity score matching was used to evaluate the average treatment effect of treated(ATT)of spousal chronic health shock. Ordinary linear square(OLS) regression was also deployed to explore the relationship between spousal chronic health shock and subjective well-being.

\section{Results} -0.209 in radius matching with caliper, and it is statistically significant; however, in rural area, ATT was 0.069, and it didn't achieve statistical significance. The results of OLS regression after PSM also suggested that spousal chronic health shock had a negative effect on subjective well-being of urban elderly, and for rural elderly, there was no such an effect.

\section{Conclusions}

21 Our study highlights more attention needs to be paid in subjective well-being of individual whose spouse suffers from chronic conditions, especially in urban China. 


\section{Introduction}

Chronic diseases including hypertension, stroke, heart disease and so on, are threating more and more families in China, especially in elderly households[1]. Chronic conditions have brought not only heavy economic burden on families, but also negative emotional and mental impacts to patients and their family members[2]. On one hand, at patient level, chronic conditions increases the health cost, physical disabilities and pain; and on the other hand, other family members especially spouse, would undertake more housework and psychological burden[3]. Researches show that there is a casual relationship between chronic health shock and spouse's self-reported health[4] and depressive symptom[3-5].

Couples who live together for a long time are closely related to each other's living environment. The change of one's condition can affect another. Therefore, chronic health shock of one family member can also affect spouse's life quality and health. First of all, becoming a caregiver would bring a heavy life burden and pressure to spouse. Secondly, due to the consistency of the living environment of both spouses, the socio-economic status of spouse would also be affected. Research has also found that the appearance of severe health problems of individual would immediately worsen spouse's health condition and may continually affect spouse's life trajectory and finally the death rate [4]. Therefore, when considering the effect of chronic health shock, spouse should be taken into consideration, especially among older couples. However, research on the effect of chronic health shock on spouse among older couples is extremely limited in China.

Subjective well-being (SWB) is an overall assessment and evaluation of individual on their current quality of life $[6,7]$. It is strongly associated with psychological and physical health[8] and could provide important guidance for making policy. Subjective well-being is closely related to health and the link could be even higher in elderly people because they can a easily be suffer from chronic diseases[9]. Previous studies have demonstrated the effects of chronic health shock on spouse's self-reported health, depressive symptom, psychological distress and retirement choice[4, $5,10,11]$. The aim of this study is to explore the effect of chronic health shock of elderly people on spouse's subjective well-being in China. China is composed of both urban and rural areas due to the particularity of system. Despite the rapid economic development and great improvement in economic and health inequality in China over the past decades, there are still considerable disparities in living conditions, income, education, health and 
1 happiness between urban and rural areas [12]. This difference in system between urban and rural areas

2 resulted in the urban-rural distinction of macro living environment for the elderly. A research using panel

3 data to study changes in the delight of Chinese urban and rural residents in 2005 and 2015 showed that

4 in both years, urban residents were happier than their rural counterparts[12]. Another research using

5 nationally representative data also demonstrated that urban residents reported significantly higher

6 subjective well-being and self-reported health [13]. China's unique socioeconomic circumstances in its

7 rural and urban areas could have an important impact on the relationship between elderly couples. A

8 prior study suggested that the urban couples have higher marital quality than rural couples, due to their

9 higher cognitive ability, income level, education level; furthermore, higher marital quality results in a positive effect on urban resident's mental health [14]. Therefore, it is understandable that there are differences in subjective well-being between urban and rural elderly. What's more, due to the uneven distribution of health resources among different areas, the prevalence of chronic diseases also differs in urban and rural areas. The prevalence of chronic diseases among the elderly in urban areas is significantly lower than that in rural areas[15]. Consequently, the impact of spouse's chronic health shock on subjective well-being among urban and rural elderly need to explored. of elderly who experienced spousal chronic health shock with that of elderly who didn't experienced spousal chronic health shock. That is, their own characteristics (that could affect SWB) of these two groups may differ and the difference may furtherly be related to which group they are in (that is to say, whether they have experienced spousal chronic health shock). Therefore, to ensure the random distribution of sample, propensity score matching (PSM) are widely adopted to evaluate average effect [16]. Propensity score matching could balance observed variables of treatment group and control group in the current database. PSM provides a comprehensive measure of the extent to which common characteristics in the treatment and control groups differ[17]. Moreover, PSM allows researchers to estimate the effect of some program without explicitly modeling the relationship between the individual characteristics and the outcome of interest. In current study, we have matched the observed variables of elderly who experienced spousal chronic health shock and who didn't in urban and rural areas respectively, according to propensity score matching procedure and furtherly evaluated the average 
1 effect of spousal chronic health shock on subjective well-being of elderly.

2 Methods

3 Data source

4 The statistical information for the current study has been collected from China Health and Nutrition

5 Survey (CHNS). CHNS survey covers the nine provinces of china that vary substantially in geography,

6 economic level, public resources and health indicators. The samples in each province were selected with

7 a multistage, random cluster process.

8 The two most recent databases of CHNS - 2011 and 2015 — were used in our study, and the total sample

9 were categorized into urban sample and rural sample. Elderly was defined as those above 60 years old; according to our purpose, those who were widowed or living alone were eliminated. Additionally,

11 elderly who lost follow-up in 2015 were not concluded in our sample.

\section{Measures}

13 Dependent Variable

14 The dependent variable in our study was self-reported subjective well-being in 2015. Subjective wellbeing predicts many life outcomes such as longevity, health, income and social skills. The SWB has been measured by question "How do you rate the quality of your life at present" in CHNS. The answer of this question has following options that are "very good", "good", "fair", "bad" or "very bad"; and is coded from 5 to 1 , respectively.

\section{Independent Variables}

Spousal chronic health shock For spousal chronic health shock, in CHNS of both 2011 and 2015, residents were asked: "Has a doctor ever asked from you that are you suffer from...(1) high blood pressure; (2) diabetes; (3) myocardial infarction; (4) stroke or transient ischemic attack; (5) cancer?" If the respondent's spouse answered No for all of these diseases in 2011 but answered Yes for any of these diseases in 2015 , then he/she was regarded that he/she has been experienced spousal chronic health shock and coded 1 ; otherwise, coded 0 . Those who experienced spousal chronic health shock were in treatment group and others were in control group.

Control variables Except for spousal chronic health shock, some other variables that could be related to subjective well-being of elderly were also concluded in our analysis[18-20]. Variable work was defined 
1 by question "Are you presently working" and was also allocated as 0 if the answer was no, and if the

2 answer was yes, then it was marked as 1 . Smoking status was also defined by answer to the question

3 "Are you currently smoking". Drinking status has been set as 1 if the respondent drunk more than twice

4 a month and noted as 0 if the respondent didn't drink twice a month. Economic level was defined as the

5 annual household income per capita. In order to facilitate our analysis; we have taken log of annual

6 household income per capita in our study. What's more, preventive health service, state of chronic

7 disease, household size and some other variables were also taken in our analysis.

$8 \quad$ Analysis strategy

$9 \quad$ Propensity score matching

10 To measure the effect of spousal chronic health shock on our outcome of interest, SWB, let $\mathrm{Y}_{\mathrm{i} 1}$ 11 indicates SWB of treatment group and $\mathrm{Y}_{\mathrm{i} 0}$ indicates SWB of control group. The average treatment effect (ATE) could be defined as the following equation.

$13 \quad \mathrm{ATE}=\mathrm{E}\left(Y_{i 1}-Y_{i 0}\right)$

14 However, due to self-selection problem, ATE calculated by equation (1) is a biased estimation of program effect. The parameter we aim to estimate here is the difference between SWB of elderly in treatment group and SWB of treated elderly if they were in control group. The estimation is so called average treatment effect on the treated (ATT). ATT is more important in program effect evaluation. Assume that $\mathrm{H}=1$ represents participants (treatment group), $\mathrm{H}=0$ represents non-participants (control group). Then ATT can be anticipated as:

$\mathrm{ATT}=\mathrm{E}\left(Y_{i 1}-Y_{i 0} \mid H_{i}=1\right)$

21 Average treatment effect on the untreated (ATU) can be defined by following equation:

$22 \quad \mathrm{ATU}=\mathrm{E}\left(Y_{i 1}-Y_{i 0} \mid H=0\right)$

23 If the experiment was done by random assignment, then ATT=ATE and ATE could be measured by the 24 outcome difference of treatment group and control group. However, in non-experimental data, researchers could get $\mathrm{E}\left(Y_{i 0} \mid H_{i}=1\right)$. Appropriate strategies need to be done to solve the missing data problem. Propensity score matching is a widely adopted method in cross-sectional data. The basic idea for PSM is that, for individual $\mathrm{i}$ in treatment group, with covariates $\boldsymbol{x}_{i}$, we can find a $\mathrm{n}$ individual $\mathrm{j}$ in 
1 control group whose covariate $\boldsymbol{x}_{j} \approx \boldsymbol{x}_{i}$. Based on Ignorability Assumption, the probability that

2 individual $\mathrm{i}$ in treatment group is the same as that individual $\mathrm{j}$ in treatment group[21]. Therefore, $Y_{j}$ can

3 represent $Y_{i 0}$. Furthermore, the treatment effect of individual i can be calculated by the following

4 equation.

$5 \quad Y_{i}-\hat{Y}_{i 0}=Y_{i}-Y_{j}$

6 In doing so, for each individual in treatment group, it can be matched by another individual in the control

7 group, so that we can measure its treatment effects. Taking the average of all the treatment effects, we

8 can get an Average Treatment Effect, which is ATE.

9 The propensity score is the probability for individual i to be in the treatment group with given $\boldsymbol{x}_{i}$. It can

10 be stated as the following equation.

$11 p\left(\boldsymbol{x}_{i}\right)=P\left(H_{i}=1 \mid \boldsymbol{x}=\boldsymbol{x}_{i}\right)$

12 In this study, following a standard propensity score matching procedure, we have firstly chosen

13 covariates $\boldsymbol{x}_{\boldsymbol{i}}$, and then we have estimated propensity score. With the propensity score, a balanced

14 matching could be realized. Standardized bias could be used to test whether $\overline{\boldsymbol{x}}_{\text {treat }}$ was similar with

$15 \overline{\boldsymbol{x}}_{\text {control }}$ after the matching. The standardized bias could be calculated by the following equation.

$16 \frac{\left|\bar{x}_{\text {treat }}-\overline{\boldsymbol{x}}_{\text {control }}\right|}{\sqrt{\left(s_{x, \text { treat }}^{2}+s_{x, \text { control }}^{2}\right) / 2}}$

17 Where $s_{x, \text { treat }}^{2}$ and $s_{x, \text { control }}^{2}$ represent the sample variance of treatment group and control group,

18 respectively.

19 ATT of matched sample could be expressed as this:

$20 \widehat{A T T}=\frac{1}{N_{1}} \sum_{i: H_{i}=1}\left(Y_{i}-\widehat{Y_{l 0}}\right)$

21 Where $N_{1}=\sum_{i} D_{i}$, which represents observations in treatment group. Similarly, ATU could be

22 expressed as this:

$23 \widehat{A T U}=\frac{1}{N_{0}} \sum_{j: H_{j}=0}\left(\widehat{Y_{J 1}}-Y_{j}\right)$

24 Where $N_{0}=\sum_{j}\left(1-D_{j}\right)$, which represents observations in control group. ATE could be expressed as 25 following: 
$1 \quad \widehat{A T E}=\frac{1}{N} \sum_{i=1}^{N}\left(\hat{Y}_{i 1}-\hat{Y}_{i 0}\right)$

2 Where $N$ represents the total sample size.

3 Matching can be realized by many ways. There is no explicit rule for which matching method to be

4 adopted[22]. Usually different ways were used and the consistent results of different matching ways can

$5 \quad$ improve the robustness of study results.

6 Regression strategy

7 In our study, we have used ordinary linear square (OLS) method to furtherly figure out the association

8 between spousal chronic health shock and SWB of elderly. Although ordered regression models are

9 usually used to conduct the analysis of ordered category variables such as SWB and OLS regression, that provides a similar estimation effectiveness as ordered regression models if the model can be set

11 accurately ${ }^{[23,24]}$. Regressions of the index of SWB are run for urban and rural samples separately. The

12 regression samples have eliminated those who failed to match in propensity score matching.

\section{Results}

14 The descriptive statistic results of selected variables were expressed in table 1 . The total sample size was 2577, with 1023 in urban area and 1554 in rural area. Observations in treatment group were 117 in urban area and 208 were in rural area; observations in control group were 906 in urban area and 1346 were in rural area.

Propensity Score Matching Results

21 Table A1 in the appendix [see Additional file 1] offered the bias reduction results by nearest neighbor matching without caliper $(\mathrm{K}=4)$ of urban and rural sample. PSM balanced the observable variables in both treatment group and control group. The quality of the match is good, because from the last two lines of table A1 we can predict that there are no statistically significant differences between two group after matching.

26 Figure A1 and Figure A2 in the appendix [see Additional file 2] furtherly exhibit the kernel density 27 distribution of urban and rural samples. Changes from (a) to (b) suggested that after matching, kernel 28 density distribution of the treatment group and control group tend to be consistent, in both urban and 
1 rural areas. The differences of kernel density distribution of two groups have been greatly reduced by

2 propensity score matching.

3 Table 2 presented our propensity score estimated for the effect of treatment on subjective well-being in

4 urban and rural areas. ATT has been calculated by nearest neighbor matching without caliper $(\mathrm{K}=4)$,

5 nearest neighbor matching with caliper ( $\mathrm{K}=4$ and caliper is set at 0.01$)$, radius matching with caliper

6 (caliper is set at 0.01), kernel matching, and spline matching. For urban area, the effect of spousal

7 chronic health shock on SWB of elderly is statistically significant by all matching methods. Apparently,

8 for urban area, the results of PSM confirm our expectation that spousal chronic health shock has a

9 negative effect on SWB of elderly. However, for the rural area, the effect was not statistically significant.

Table 2 Propensity score estimated for the effect of treatment on SWB

\begin{tabular}{|c|c|c|c|c|c|}
\hline & & Treatment & Control & ATT & S.E. \\
\hline \multicolumn{6}{|l|}{ Urban } \\
\hline & $\begin{array}{l}\text { Nearest neighbor matching without } \\
\text { caliper }(\mathrm{K}=4)\end{array}$ & 3.700 & 3.884 & $-0.184 *$ & 0.102 \\
\hline & $\begin{array}{l}\text { Nearest neighbor matching with } \\
\text { caliper }(\mathrm{K}=4)\end{array}$ & 3.700 & 3.888 & $-0.189 *$ & 0.102 \\
\hline & Radius matching with caliper & 3.700 & 3.908 & $-0.209 * *$ & 0.095 \\
\hline & Kernel matching & 3.700 & 3.904 & $-0.205^{* *}$ & 0.094 \\
\hline & Spline matching & 3.700 & 3.894 & $-0.195 * *$ & 0.096 \\
\hline \multicolumn{6}{|l|}{ Rural } \\
\hline & $\begin{array}{l}\text { Nearest neighbor matching without } \\
\text { caliper }(\mathrm{K}=4)\end{array}$ & 3.744 & 3.643 & 0.100 & 0.094 \\
\hline & $\begin{array}{l}\text { Nearest neighbor matching with } \\
\text { caliper }(\mathrm{K}=4)\end{array}$ & 3.744 & 0.637 & 0.107 & 0.094 \\
\hline & Radius matching with caliper & 3.744 & 3.675 & 0.069 & 0.087 \\
\hline & Kernel matching & 3.744 & 3.698 & 0.046 & 0.086 \\
\hline & Spline matching & 3.744 & 3.694 & 0.050 & 0.084 \\
\hline
\end{tabular}

$11 \quad *_{p}<0.1, * * p<0.05, * * * p<0.01$

12 Figure 1 confirmed the OLS regression results of urban sample after matching. Sample size became

13 smaller after PSM and observations in urban were 801. From figure 1 we can perceive that spousal

14 chronic health shock has a negative effect on SWB of elderly. Besides, women have higher SWB than

15 men. Compared with those whose highest education level is junior high school and below, elderly who

16 are higher educated have higher SWB. Additionally, economic level and household members are

17 positively associated with SWB. Personal chronic condition has been negatively correlated with SWB 
of elderly.

[Insert Figure 1 here]

Figure 2 illustrated the OLS regression results of rural sample after matching. The total Observations were 968. Figure 2 also explained that spousal chronic health shock hasn't been observed to have any effect on SWB of elderly. For other control variables, elderly with higher education level have higher SWB. Economic level has been also positively associated with SWB. Drinking status also have positive association with SWB in the rural area.

[Insert Figure 2 here]

\section{Discussion}

Extensive literatures have given an idea about the linkages between spousal illness and personal physical or mental health [3, 25-27]. Despite the substantial evidence, an important index for individual's life quality -subjective well-being hasn't been focused. China, with the largest population of the world, hasn't been concerned before, either. Little is known about how spousal chronic health shock influenced individual's SWB, especially in China and whether this differs in urban and rural area. In this study, by using a nationally representative survey data of China, we have scrutinized the effect of spousal chronic health shock on subjective well-being of elderly in both urban and rural areas. This current study advances our knowledge of the causal effect using method of propensity score matching. First, our study demonstrated that the onset of spousal chronic diseases would worsen the SWB of elderly in urban China. We explain several ways of influence mechanism. Firstly, Chronic illnesses has brought heavy economic burden to individual and family. Those families who have been suffering from chronic diseases are facing with heavy catastrophic health expenditures(CHE)[28]. Economic condition can strongly affect SWB, especially in low- and middle-income countries like China[29]. Suffering from chronic illness usually means family's suffering from a sudden income reduction and this condition could worsen SWB of elderly. Secondly, for older couples, if one of them got chronic illness, the other usually needs to play the role of a caregiver. Over one-third of all caregivers were proved to be spouses and approximately three fourths of them were over the age of 65[11]. In China, "aging at home" is the most popular and traditional way of old-aged pension[30]. What's more, there are more and more empty- 
1 related to care, for the chronically ailing spouse. However, being a caregiver also bring several burdens

2 to elderly. Caregivers need to take on many different types of tasks. For example, caregivers need to

3 assist their ailing spouse with enough daily activities, monitor their diets and provide care after a

4 debilitating illness. A large body of literature has documented that caregivers tend to report worse

5 physical health and higher rates of depression and anxiety, especially among the elderly[32-35]. As a

6 consequence, a potential explanation for our result that spousal chronic health shock has a negative

7 effect on elderly's SWB is that elderly whose spouse suffered from chronic health shock may need to

8 provide care for their spouse and being a caregiver brings both physical and mental burden to their daily

9 life and consequently, their attitude and satisfaction to current life decreases. Thirdly, looking after mutually in couple's plays a crucial part in life of old age. Elderly couples support each other by accompanying in hardship of life, comforting and protecting each other. If one suddenly suffers from chronic disease, his/her daily life would be affected, so would his/her spouse. Not only spousal chronic condition brings economic burden, but also social support and family support will also be damaged. Emotion sustenance is very important for elderly, especially in urban China, where elder couples usually live a better life because of higher economic level[36]. However, if spouse suffers from chronic disease, for elderly, spouse's concern about their daily life may decrease. The change of life style and emotion sustenance may worsen subjective well-being of elderly. The results of our study from urban and rural dimensions also have been suggested the different role spousal chronic health shock played in SWB of elderly. Our results proved that spousal chronic health shock significantly worsens the SWB of urban elderly, but the effect for rural elderly isn't statistically significant. Previous studies about subjective well-being could provide reasons for this. It is widely recognized that people with higher education level are more sensitive to happiness[36, 37]. Compared with rural residents, urban residents usually have higher level of education. Our descriptive results also presented that urban residents are better educated. With less well educated than urban elderly, rural elderly may concentrate more on financial support and have lower consciousness and understanding level of happiness or SWB[38]. Besides, rural elderly usually have more ways to participate in working than urban elderly. Labor participation plays an important role in SWB. Previous literatures have documented that participation in work could significantly improve individual's SWB[39]. This 
1 relationship outlines that labor participation could relief elder's stress when his/her spouse suffer from

2 chronic illness. Another potential explanation for urban-rural disparity is that urban households in China

3 usually consist of less members than rural households. Our descriptive results have also proven that

4 rural household size is larger than urban. Small household makes elderly relies more on their spouse but

5 not children or other relatives. Additionally, people living in rural usually have stronger neighborhood

6 relationship. Consequently, the elderly living in rural China would harder to be influenced by spousal

$7 \quad$ chronic health shock.

\section{Conclusions}

9 Chronic diseases are becoming more and more popular in China, especially among elderly. Our study found that spousal chronic health shock would have a significant negative effect on elderly's subjective wellbeing in urban China. We can explain this relationship in three ways. First, the onset of chronic diseases has brought heavy economic burden to a family and SWB of elderly is relatively associated with economic level. Second, spousal suffering from chronic disease usually means elderly need to become a caregiver and this will bring living burden to him/her. Third, for urban elderly couples with closer marital relationship, if one partner suffers from chronic illness, the other may be lonely and lack of emotional support. In rural China, there was no effect has been found between spousal chronic health shock and elderly's SWB. It is understandable because rural residents have lower educational level and thus they are less sensitive to subjective well-being. Furthermore, rural elderly usually have more ways to participate in labor activities and more family members and friends, which could compensate SWB reduction caused by spousal chronic health shock. This study firstly evaluated the effect of spousal chronic health shock of SWB of elderly from rural and urban dimensions in China. Our results suggest that more attention need to be payed to the elderly whose spouse are suffering from chronic illness, especially in urban area.

There are also several limitations in our analysis. We proposed several possible explanations. However, the influence mechanism of this effect needs to be further explored in future studies. In our study we have concentrated on whether spouse catches up chronic illness, while other studies suggested that different numbers of chronic conditions also has a different effect on elderly's SWB[5]. Future research should consider different numbers of chronic illness. Lastly, in our study, we didn't consider the 
1 difference of different diseases. The effect of different chronic diseases, such as hypertension and cancer,

2 also need to be explored in future.

4 Declarations:

- Ethics approval and consent to participate:

The ethics approval was obtained by the review board from the University of North Carolina at Chapel Hill, National Institute for Nutrition and Food Safety, China Center for Disease Control and Prevention and China-Japan Friendship Hospital. Informed consent was obtained, and data were anonymized for the analysis.

- Consent for publication:

$11 \quad$ Not applicable.

- Availability of data and materials:

3 The datasets generated and analysed during the current study were derived from the China Health and Nutrition Survey (CHNS). They are opened to everyone. Researchers who want to use these data can visit http://www.cpc.unc.edu/projects/china.).

\section{- Competing interests:}

17 The authors declare that they have no competing interests.

\section{- Funding:}

9 This study was funded by the Project of Shaanxi Social Science Foundation (2017S024) and National Natural Science Foundation of China (71874137).

\section{- Authors' contributions:}

ZZ, DC processed the data and were major contributors in writing the manuscript. YZ and DZ participated in the design this study. YR and CS acquired data and provided administrative support for data analysis. RN, GL and XZ was involved in revising the manuscript critically for important intellectual content. XF, JZ and JG offered suggestions to complete this study and made substantial contributions to revised this article. All authors have read and approved the final manuscript.

- Acknowledgments:

The authors wish to thank the supported provided by the Youth Innovation Team of Shaanxi 
Universities. Highly tribute shall be paid to Mr. Bo Li, for his great support and considerable time and effort on the comments of this paper.

This research uses data from China Health and Nutrition Survey (CHNS). We thank the National Institute for Nutrition and Health, China Center for Disease Control and Prevention, Carolina Population Center (P2C HD050924, T32 HD007168), the University of North Carolina at Chapel Hill, the NIH (R01-HD30880, DK056350, R24 HD050924, and R01-HD38700) and the NIH

\section{References}

13 1. Wang $\mathrm{Z}, \mathrm{Li} \mathrm{X}$, Chen M: Catastrophic health expenditures and its inequality in elderly

6. Wang P, VanderWeele TJJSir: Empirical research on factors related to the subjective well-

4. Valle G, Weeks JA, Taylor MG, Eberstein IW: Mental and physical health consequences of

3. Min J, Yorgason JB, Fast J, Chudyk A: The Impact of Spouse's Illness on Depressive

2. Hollingshaus MS, Utz RL: Depressive symptoms following the diagnosis of major chronic illness. Society and Mental Health 2013, 3(1):22-39. 9 Symptoms: The Roles of Spousal Caregiving and Marital Satisfaction. The Journals of Gerontology: Series B 2019. being of Chinese urban residents. 2011, 101(3):447-459.

7. Veenhoven RJSir: Is happiness relative? 1991, 24(1):1-34. 
1 8. Deng J, Hu J, Wu W, Dong B, Wu HJIjogp: Subjective well-being, social support, and agerelated functioning among the very old in China. 2010, 25(7):697-703.

3 9. Steptoe A, Deaton A, Stone AAJTL: Subjective wellbeing, health, and ageing. 2015, 385(9968):640-648.

10. Hagedoorn M, Sanderman R, Ranchor AV, Brilman EI, Kempen GI, Ormel JJJopr: Chronic disease in elderly couples: are women more responsive to their spouses' health condition

15. Xuejie B, Yusi C: Differences in Regional Medical Resources Allocation, Medical Condition, and Individual Health(in Chinese). Journal of Xi'an Jiaotong University(Social science) 2019, 39(02):86-95.

16. Trujillo AJ, Portillo JE, Vernon JAJIjohcf, economics: The impact of subsidized health insurance for the poor: evaluating the Colombian experience using propensity score matching. 2005, 5(3):211-239.

17. Dehejia RH, Wahba SJJotAsA: Causal effects in nonexperimental studies: Reevaluating the evaluation of training programs. 1999, 94(448):1053-1062.

18. Brown PH, Tierney BJTJoS-E: Religion and subjective well-being among the elderly in China. 2009, 38(2):310-319.

19. Wang XJJohp: Subjective well-being associated with size of social network and social 
support of elderly. 2016, 21(6):1037-1042.

20. $\mathrm{Hu} \mathrm{H}, \mathrm{Lu} \mathrm{Y}$ : Income Inequality, Health and Subjective Well-being among the Elderly: Empirical Evidence on the Aging of China(in Chinese). China Soft Science 2012(11):41-56.

21. Wooldridge JMJRiE: Should instrumental variables be used as matching variables? 2016, 70(2):232-237.

22. Chen Q: Advanced Econometrics(in Chinese): Higher education press; 2010.

23. Ferrer-i-Carbonell A, Frijters PJTEJ: How important is methodology for the estimates of the determinants of happiness? 2004, 114(497):641-659.

24. Ferrer-i-Carbonell AJJope: Income and well-being: an empirical analysis of the comparison income effect. 2005, 89(5-6):997-1019.

25. Revenson TA, Deborah Majerovitz SJA, Rheumatology ROJotACo: The effects of chronic illness on the spouse. Social resources as stress buffers. 1991, 4(2):63-72.

26. Polenick CA, Birditt KS, Turkelson A, Kales HCJAoBM: Individual-level and couple-level discordant chronic conditions: Longitudinal links to functional disability. 2020, 54(7):455469.

27. Polenick CA, Birditt KS, Turkelson A, Bugajski BC, Kales HCJTJoGSB: Discordant chronic conditions and depressive symptoms: Longitudinal associations among middle-aged and older couples. 2019.

28. Wang Z, Li X, Chen MJIjfeih: Catastrophic health expenditures and its inequality in elderly households with chronic disease patients in China. 2015, 14(1):8.

29. Howell RT, Howell CJJPb: The relation of economic status to subjective well-being in developing countries: A meta-analysis. 2008, 134(4):536.

30. Du S, Zhang Y, Feng Z, Feng Z: Current situation and Countermeasures of urban home based care(in Chinese). Chinese Journal of Gerontology 2013, 033(012):2980-2982.

31. Su H, Cao J, Zhou Y, Wang L, Xing LJAog, geriatrics: The mediating effect of coping style on personality and mental health among elderly Chinese empty-nester: A cross-sectional study. 2018, 75:197-201.

32. Ho SC, Chan A, Woo J, Chong P, Sham AJJoGSABS, Sciences M: Impact of caregiving on 
health and quality of life: a comparative population-based study of caregivers for elderly persons and noncaregivers. 2009, 64(8):873-879.

33. Hou RJ, Wong SY-S, Yip BH-K, Hung AT, Lo HH-M, Chan PH, Lo CS, Kwok TC-Y, Tang WK, Mak WWJP et al: The effects of mindfulness-based stress reduction program on the mental health of family caregivers: a randomized controlled trial. 2014, 83(1):45-53.

34. Denno MS, Gillard PJ, Graham GD, DiBonaventura MD, Goren A, Varon SF, Zorowitz RJAopm, rehabilitation: Anxiety and depression associated with caregiver burden in caregivers of stroke survivors with spasticity. 2013, 94(9):1731-1736.

35. Yang X, Hao Y, George SM, Wang LJH, Outcomes QoL: Factors associated with healthrelated quality of life among Chinese caregivers of the older adults living in the community: a cross-sectional study. 2012, 10(1):143.

36. Xin-feng C, Quan-bao J: Widowhood and the Subjective Well-being of Elderly:Analysis of Gender and Location Difference(in Chinese). POPULATION AND DEVELOPMENT 2017(4).

37. Yu Y: How Education Affects Happiness: Research Progress on Education, Public Expenditure on Education, and Subjective Well-being(in Chinese). Peking University Education Review 2014, 12(3):105-120.

38. Chen Q, Chi G: Social Comparisons, Inclusion and Happiness:An Empirical Evidence from Rural China(in Chinese). Economic Review 2014, 000(4):3-16.

39. Sage DJJoHS: Do active labour market policies promote the subjective well-being of the unemployed? Evidence from the UK National Well-Being Programme. 2015, 16(5):12811298.

Figure legends

Figure 1, OLS regression after PSM - urban sample, shows the OLS regression result after matching treatment and control group in urban sample. The far right column shows the coefficient and its $95 \%$ confidence interval. With controlling other variables, the negative effect of spousal chronic health shock is statistically significant. 
1 Figure 2, OLS regression after PSM - rural sample, shows the OLS regression result after matching

2 treatment and control group in rural sample. The far right column shows the coefficient and its $95 \%$

3 confidence interval. The coefficient isn't statistically significant, which is there is no effect found here.

$4 \quad$ Additional Files

5 Additional file 1.docx: Table A1, bias reduction results by nearest neighbor matching;

6 Additional file 2.docx: Figure A1, kernel density distribution - urban sample; Figure A2, kernel

7 density distribution - rural sample. 
ID

$\operatorname{coef}(95 \% \mathrm{Cl})$

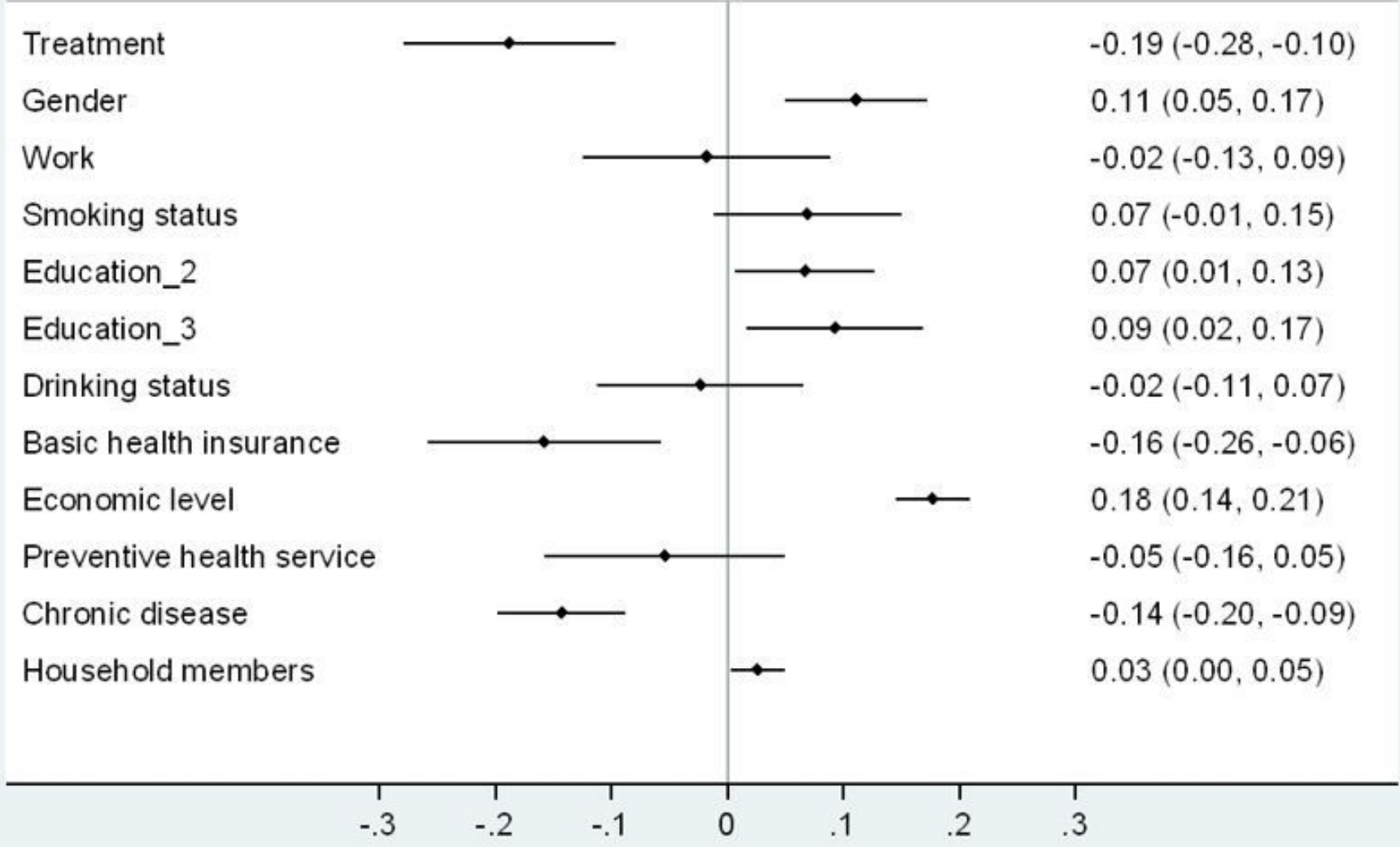

Figure 1

OLS regression after PSM - urban sample, shows the OLS regression result after matching treatment and control group in urban sample. The far right column shows the coefficient and its $95 \%$ confidence interval. With controlling other variables, the negative effect of spousal chronic health shock is statistically significant. 


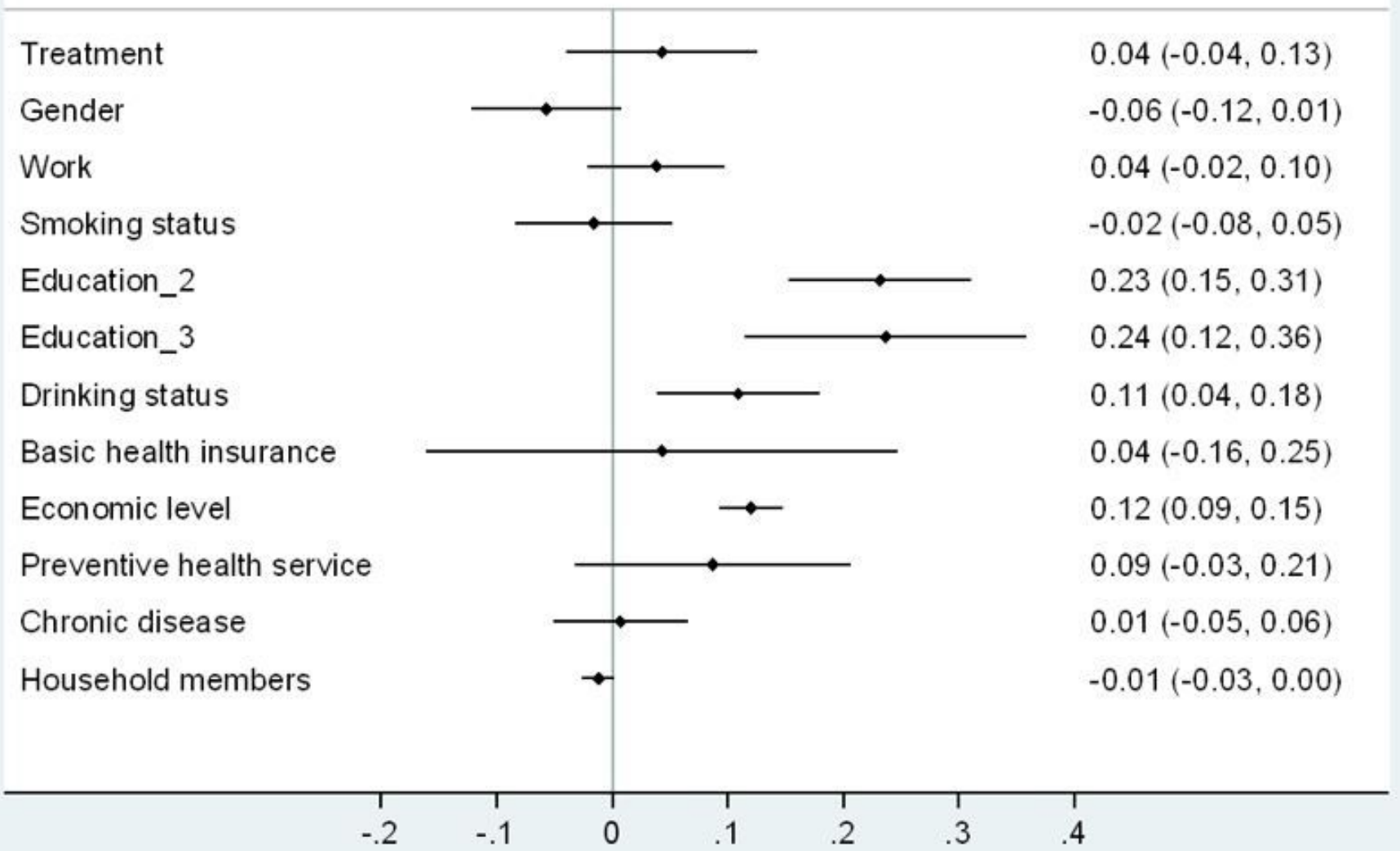

Figure 2

OLS regression after PSM - rural sample, shows the OLS regression result after matching treatment and control group in rural sample. The far right column shows the coefficient and its $95 \%$ confidence interval. The coefficient isn't statistically significant, which is there is no effect found here.

\section{Supplementary Files}

This is a list of supplementary files associated with this preprint. Click to download.

- AdditionalFiles.docx

- Additionalfile1.docx

- Additionalfile2.docx 